
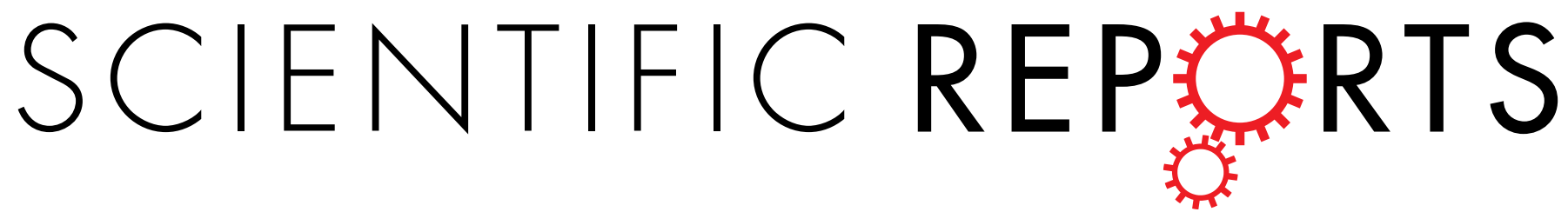

\title{
OPEN
}

\section{Serum lysophospholipid levels are altered in dyslipidemic hamsters}

\author{
Susana Suárez-García ${ }^{1}$, Antoni Caimari ${ }^{2}$, Josep Maria del Bas ${ }^{2}$, Manuel Suárez ${ }^{1}$ \& Lluís Arola ${ }^{1,2}$
}

Received: 26 January 2017

Dyslipidemias are common disorders that predispose individuals to severe diseases. It is known that healthy living habits can prevent dyslipidemias if they are diagnosed properly. Therefore, biomarkers that assist in diagnosis are essential. The aim of this study was to identify biomarkers of dyslipidemia progression, which in turn disclose its etiology. These findings will pave the way for examinations of the regulatory mechanisms involved in dyslipidemias. Hamsters were fed either a normal-fat diet (NFD) or a high-fat diet. Some of the NFD-fed animals were further treated with the hyperlipidemic agent Poloxamer 407. Non-targeted metabolomics was used to investigate progressive changes in unknown serum metabolites. The hepatic expression of putative biomarker-related genes was also analyzed. The serum levels of lysophospholipids (Lyso-PLs) and their related enzymes lecithincholesterol acyltransferase (LCAT), secreted phospholipase $A_{2}\left(\right.$ SPLA $_{2}$ ) and paraoxonase-1 were altered in dyslipidemic hamsters. Lysophosphatidylcholine levels were increased in diet-induced dyslipidemic groups, whereas lysophosphatidylethanolamine levels increased in response to the chemical treatment. The liver was significantly involved in regulating the levels of these molecules, based on the modified expression of endothelial lipase ( $L i p g), \mathrm{sPLA}_{2}$ (Pla2g2a) and acyltransferases (Lcat and Lpcat3). We concluded that Lyso-PL evaluation could aid in the comprehensive diagnosis and management of lipid disorders.

Dyslipidemias are disturbances in lipid metabolism that have a high prevalence in both developed and developing countries and lead to changes in the levels and/or functions of the plasma lipoproteins ${ }^{1-3}$. These lipid disorders can appear as an elevation in the levels of circulating triglycerides, total cholesterol (TC) and low-density lipoprotein cholesterol (LDLc), as well as a reduction in high-density lipoprotein cholesterol (HDLc) levels ${ }^{4}$. Alterations in lipid metabolism and transport can have severe implications, predisposing the patients to the development of cardiovascular diseases, fatty liver disorders and different types of cancer $^{5-8}$.

In terms of the etiology, the development of dyslipidemia in humans depends on both genetic and environmental factors. Thus, lipid management begins with the exclusion of secondary causes of metabolic disorder ${ }^{9}$. A diet rich in saturated fats and cholesterol is a well-established source of acquired dyslipidemias ${ }^{4-6}$, but accumulating evidence has shown that certain exogenous compounds, such as alcohol, tobacco or some drugs, also induce lipid disorders when consumed regularly over a long period of time ${ }^{9-11}$. Advances in our understanding of the mechanisms involved in dyslipidemia progression that depend on the type of triggering factor are necessary to improve the diagnosis and treatment of dyslipidemia-related diseases.

The Golden Syrian hamster is the rodent that displays greatest similarity to humans regarding lipoprotein metabolism ${ }^{12}$. In the present work, this hamster model was selected to investigate the biological alterations that occur after the chronic intake of a high-fat diet (HFD) for a maximum of 30 days. Furthermore, an additional group of animals was treated with the hyperlipidemic agent Poloxamer 407 (P407) to generate a different form of dyslipidemia that was not induced by diet. P407 is a hydrophilic, non-ionic, surface-active compound with low toxicity that has been extensively used in pharmaceutical preparations and in common personal care products, such as toothpastes and cosmetics ${ }^{13}$. After parental administration, P407 induces hypertriglyceridemia and hypercholesterolemia in rodents by inhibiting the heparin-releasable fraction of lipoprotein lipase (LPL) and the cholesterol $7 \alpha$-hydroxylase $(\mathrm{C} 7 \alpha \mathrm{H})$, respectively ${ }^{14,15}$. However, chronic treatment with $\mathrm{P} 407$ does not seem to stimulate 3-hydroxy-3-methylglutaryl coenzyme A (HMG-CoA) reductase, a key enzyme involved in the synthesis of cholesterol, target of many anti-dyslipidemic drugs ${ }^{16}$. P407-induced hyperlipidemia, which exhibits more pronounced plasma triglyceride levels than cholesterol levels, is dose-dependent and remains at steady levels with

${ }^{1}$ Nutrigenomics Research Group, Department of Biochemistry and Biotechnology, Universitat Rovira i Virgili (URV), Tarragona, 43007, Spain. ${ }^{2}$ Technological Unit of Nutrition and Health. EURECAT-Technological Center of Catalonia, Reus, 43204, Spain. Correspondence and requests for materials should be addressed to M.S. (email: manuel. suarez@urv.cat) 
repetitive injections of the drug approximately every 3 days ${ }^{17}$. Thus, the P407-treated rodent and the HFD-fed hamster are well-established models of atherogenesis that properly reproduce the adverse events that precede the development of atherosclerotic lesions in humans ${ }^{18,19}$.

In this context, the use of an omics-based strategy allowed us to provide a global characterization of the changes in the circulating metabolome associated with the long-term administration of both pro-dyslipidemic treatments, as well as to investigate the main hepatic mechanisms. Some studies have been performed in HFD-fed hamsters ${ }^{20-22}$ and dyslipidemic subjects ${ }^{23,24}$ using non-targeted metabolomics techniques, but, to date, research on P407-treated rodents has not utilized this approach. All these studies point to the importance of comprehensive investigations for expanding our understanding of the complex networks involved in the development of lipid disorders and the identification of novel biomarkers for the early diagnosis and management of related diseases.

Therefore, the main objective of the present study was to identify non-invasive biomarkers of dyslipidemia progression that reveal the etiology of the disorder. Therefore, we used ultra-high-performance liquid chromatography coupled to quadrupole time-of-flight mass spectrometry (UHPLC-Q-TOF-MS/MS) to evaluate the alterations in the serum metabolite levels of adult hamsters in response to different periods of HFD feeding and treatment with P407. Furthermore, after the untargeted identification of the metabolites associated with each type of dyslipidemia, the hepatic mechanisms involved in the metabolism of these molecules were also examined to extend our knowledge of the modes of action of the two treatments and their disparities.

\section{Material and Methods}

Chemicals. Methanol (Scharlab S.L., Barcelona, Spain) and glacial acetic acid (Panreac, Barcelona, Spain) were of high-performance liquid chromatography (HPLC) analytical grade. Ultrapure water was obtained from a Milli-Q advantage A10 system (Madrid, Spain). Phenylalanin ${ }^{13} \mathrm{C}$ (Fluka/Sigma-Aldrich, Madrid, Spain) was used as an internal standard (IS) for the untargeted metabolomics analysis ${ }^{25}$. The standard was dissolved in methanol at $1 \mathrm{mg} / \mathrm{mL}$ and stored at $-20^{\circ} \mathrm{C}$ prior to use. Butylated hydroxytoluene (BHT, Fluka/Sigma-Aldrich, Madrid, Spain) was added to the extraction solution to avoid metabolite oxidation during the sample extraction. P407 (Fluka/Sigma-Aldrich, Madrid, Spain) was administered to the animals to induce dyslipidemia.

Animal studies. Two different in vivo studies were performed to identify serum biomarkers of dyslipidemia. All of procedures were approved by the Animal Ethics Committee of the University Rovira i Virgili (Tarragona, Spain) and they have been performed in accordance with the European Communities Council Directive (86/609/ EEC).

A first exploratory study was performed using 3-month-old male Golden Syrian hamsters (Charles River Laboratories, Barcelona, Spain). Animals weighing $130 \mathrm{~g}$ were housed singly at $22^{\circ} \mathrm{C}$ with a light/dark period of $12 \mathrm{~h}$ (lights on at 09:00 h) and with free access to food and water. After an adaptation period of 4 days, the hamsters were randomly distributed into two experimental groups $(\mathrm{n}=16$ per group $)$ and fed either a normal-fat diet (NFD; D10051906) or a high-fat diet (HFD; D10051907) (Research Diets Inc., New Brunswick, NJ, USA) ad libitum. The composition of the diets is shown in Supplementary Table S1. The NFD provided 10\% energy as fat, whereas the HFD provided $21 \%$ energy as fat because of the high content of lard. Eight animals of each group were sacrificed on day $15(15 \mathrm{~d})$ and the other 8 on day $30(30 \mathrm{~d})$.

A second study was conducted using a different strategy to induce dyslipidemia. For this purpose, 8-week-old male Golden Syrian hamsters (Janvier, Le Genest-St-Isle, France) weighing $110 \mathrm{~g}$ were housed individually in cages at $22^{\circ} \mathrm{C}$ with a light/dark period of $12 \mathrm{~h}$ and free access to food and water. After an adaptation period of 2 weeks in which animals were fed an NFD, they were randomly assigned to six groups ( $\mathrm{n}=9-10$ per group). Three groups continued the experiment for 4 days (4d) to detect early changes in the metabolome profile between groups, whereas the other three groups were sacrificed at $30 \mathrm{~d}$. The experimental design is described below. Two groups were maintained on the NFD and served as control animals (C-4d and C-30d groups), two other groups were fed the lard-based HFD (HFD-4d and HFD-30d groups) and the last two groups were fed the NFD and periodically injected with $50 \mathrm{mg}$ of $\mathrm{P} 407$ per kg body weight every $72 \mathrm{~h}$ to induce dyslipidemia (P407-4d and P407-30d groups). Until now, P407 has been extensively used in mice ${ }^{15,26}$ and rats $^{14,27,28}$ but, to the best of our knowledge, only one study has been conducted in Golden Syrian hamsters that were periodically injected with P407 to induce dose-dependent hyperlipidemia ${ }^{29}$. In the present work, we have utilized a lower dose of P407 than the dose used by Liu et al. ${ }^{29}$. Based on preliminary studies, this quantity was selected as the adequate dose to achieve the development of moderate hypertriglyceridemia and hypercholesterolemia in hamsters (see Supplementary Fig. S1). The animals that were not treated with $\mathrm{P} 407$ received injections of vehicle $(0.9 \% \mathrm{NaCl})$ with equal frequency. The hamsters were sacrificed $24 \mathrm{~h}$ after the last administration of P407 or vehicle. Lean and fat mass measurements (in grams) were performed without anesthesia on the first and the last days of the study using an EchoMRI-700 ${ }^{\mathrm{TM}}$ (Echo Medical Systems, LLC., TX, USA).

In both experiments, the hamsters were deprived of food for $6 \mathrm{~h}$ on the day of sacrifice and euthanized under anesthesia (pentobarbital sodium, $80 \mathrm{mg}$ per $\mathrm{kg}$ body weight). Blood was collected by cardiac puncture and serum was obtained by centrifugation $(2,000 \mathrm{~g}$ for $15 \mathrm{~min})$. The livers were dissected, weighted and immediately frozen in liquid nitrogen. All the samples were stored at $-80^{\circ} \mathrm{C}$ until further analyses.

Serum measurements. Enzymatic colorimetric kits were used for the determination of glucose and triglycerides (QCA, Barcelona, Spain), phospholipids (Spinreact, Girona, Spain), non-esterified free fatty acids (NEFAs) (WAKO, Neuss, Germany), TC, HDLc and LDLc (Bioassay systems, Hayward, CA, USA). The circulating levels of the enzymes lecithin-cholesterol acyltransferase (LCAT), group IIA secreted phospholipase $\mathrm{A}_{2}$ ( $\mathrm{sLA}_{2}$-IIA), and paraoxonase-1 (PON1) were measured with hamster ELISA kits (MyBioSource, San Diego, CA, USA). 
Sample preparation for non-targeted metabolomics. Serum extracts were prepared using a procedure similar to the method described by Jové et al. ${ }^{20}$. Briefly, $90 \mu \mathrm{L}$ of cold methanol containing phenylalanine ${ }^{13} \mathrm{C}$ as the IS $(10 \mathrm{ppm})$ and BHT $(1 \mu \mathrm{M})$ as an antioxidant were added to $30 \mu \mathrm{L}$ of serum, vortexed for 1 minute and incubated at $-20^{\circ} \mathrm{C}$ for $1 \mathrm{~h}$ to precipitate proteins. Samples were centrifuged at $12,000 \mathrm{~g}$ for $5 \mathrm{~min}$ at $4{ }^{\circ} \mathrm{C}$ and the supernatants were collected and dried in a SpeedVac (Thermo Fisher Scientific, Waltham, MA, USA). Samples were re-suspended in $100 \mu \mathrm{L}$ of Milli-Q water containing $0.2 \%$ acetic acid:methanol $0.2 \%$ acetic acid (1:1) prior to injection.

LC-MS and LC-MS/MS analyses. Both untargeted and targeted analyses were performed on serum extracts using an UHPLC 1290 coupled to a Q-TOF 6550 mass spectrometer (Agilent Technologies, Palo Alto, CA, USA). The temperature in the autosampler was maintained at $4{ }^{\circ} \mathrm{C}$ throughout the analysis, and the samples were randomized.

Solvent A consisted of $0.2 \%$ acetic acid and solvent B consisted of methanol with $0.2 \%$ acetic acid. Two microliters of sample were applied to a Zorbax SB-Aq ( $1.8 \mu \mathrm{m}$ particle size, $2.1 \mathrm{~mm}$ internal diameter $\times 50 \mathrm{~mm}$ length) analytical column maintained at $60^{\circ} \mathrm{C}$ and equipped with a Zorbax SB-C8 $(3.5 \mu \mathrm{m}, 2.1 \times 30 \mathrm{~mm})$ guard column, also from Agilent Technologies. Chromatographic separation was performed by continuous gradient elution at flow rate of $0.6 \mathrm{~mL} / \mathrm{min}$ starting at $2 \% \mathrm{~B}$ and increasing to $98 \% \mathrm{~B}$ in $13 \mathrm{~min}$, where it was maintained isocratically for $6 \mathrm{~min}$. The chromatographic system was returned to the initial conditions in $1 \mathrm{~min}$, followed by a 5 -min equilibration prior to the subsequent injection. Ionization in the mass spectrometer was performed by AJS ESI operated either in positive (+ESI) and negative (-ESI) mode with the following settings: nebulizer gas, nitrogen at a pressure of $45 \mathrm{psi}$; desolvation gas flow rate, $9 \mathrm{~L} / \mathrm{min}$ at $325^{\circ} \mathrm{C}$; source temperature and gas flow rate, $150^{\circ} \mathrm{C}$ and $12 \mathrm{~L} /$ $\mathrm{min}$, respectively; capillary voltage, $4 \mathrm{kV}$; fragmentor, $125 \mathrm{~V}$. Accurate LC-MS mass spectra were acquired over the $40-1600 \mathrm{~m} / z$ range at a scan rate of $1.5 \mathrm{spectra} / \mathrm{s}$. A reference solution was used for continuous calibration with the following reference masses: 121.0509 and $922.0098 \mathrm{~m} / z$ for + ESI and 119.0363 and $980.0164 \mathrm{~m} / z$ for - ESI. Quality controls (QC) were prepared mixing $10 \mu \mathrm{L}$ of each serum extract. At the start of the run, eight QC were injected for column conditioning and then every ten samples to assess instrument stability. For targeted analyses, the instrument was operating in MS and MS/MS modes, and the other analytical conditions were the same as described above. The MS/MS spectra of the metabolites were obtained at collision energies of 10 and $20 \mathrm{eV}$.

Data processing and metabolite identification. All the software programs were provided by Agilent Technologies (Palo Alto, CA, USA). MassHunter Data Acquisition software was used to generate profile peak data from the spectra and MassHunter Qualitative Analysis software was used to obtain the molecular features of the samples. The peaks were aligned using Mass Profiler Professional (MPP) and mass and retention time (RT) windows of $0.1 \% \pm 0.15 \mathrm{~min}$ and $15.0 \mathrm{ppm} \pm 2.0 \mathrm{mDa}$, respectively. Each chemical entity obtained from MPP is associated with a particular neutral mass, RT and abundance value. The list of molecular entities was filtered by selecting only those entities that were present in at least $80 \%$ of the samples in the same group. The normalized abundances were obtained by performing a base- 2 logarithmic transformation of the ratio of each analyte to the IS. Candidate biomarkers were first identified using metabolite databases $\left(\mathrm{METLIN}^{30}, \mathrm{HMDB}^{31}\right.$, and LIPID MAPS $^{32}$ ). Finally, the structural information about the molecules of interest was provided by the fragmentation data obtained at different collision energies.

Total RNA isolation and gene expression analysis. Livers used for mRNA analysis were homogenized and total RNA was extracted with TRIzol Reagent and purified on RNeasy Mini Kit spin columns (Qiagen, Barcelona, Spain) according to the manufacturer's protocols. RNA yield was quantified on a Nanodrop 1000 Spectrophotometer (Thermo Scientific, Madrid, Spain) and tested for purity by measuring A260/280 ratio. Total RNA $(0.5 \mu \mathrm{g}$; in a final volume of $20 \mu \mathrm{L})$ was reverse transcribed into complementary DNA (cDNA) using the High-Capacity cDNA Reverse Transcription Kit (Applied Biosystems, Madrid, Spain). cDNAs were subjected to quantitative real-time reverse transcriptase-polymerase chain reaction (qRT-PCR) amplification using SYBR Green PCR Master Mix (Bio-Rad, Barcelona, Spain). All reactions were performed in triplicate. When possible, primers were designed to span an exon-exon junction at the conserved domain of the gene of interest, avoiding the problem of the possible contamination by genomic DNA. Primer sequences for the target genes are listed in Supplementary Table S2 and were obtained from Biomers.net (Ulm, Germany). The relative mRNA expression levels were calculated using the $2^{-\Delta \Delta \mathrm{Ct}}$ method $^{33}$ with $\beta$-actin as the reference gene and were normalized to the C-4d group.

Statistical analysis. The results are presented as the means \pm SEM from the indicated number of hamsters. The assumption of normality was determined using the Shapiro-Wilk test, and the homoscedasticity between groups was assessed using Levene's test. Once these conditions were verified, differences among groups were assessed using two-way ANOVA to evaluate the main effects of the time (duration of the experiment) and the pro-dyslipidemic intervention (HFD and P407) and their interaction. For the untargeted analysis, the p-values were calculated using the Benjamini-Hochberg correction. When any of the effects was statistically significant, one-way ANOVA was used to determine the differences among all the means at once. Tukey's post hoc test was applied when the variances were similar and the Games-Howell test was applied if this assumption was not fulfilled. Student's t-test was used for single statistical comparisons. A two-tailed value of $\mathrm{p}<0.05$ was considered statistically significant. All statistical analyses were performed with Statistical Package for Social Sciences (IBM SPSS Statistics, version 19.0).

Results

Identification of a class of lipids involved in dyslipidemia progression. Based on the results from the initial study, the development of dyslipidemia was successfully induced by the chronic administration of the 
Body weight

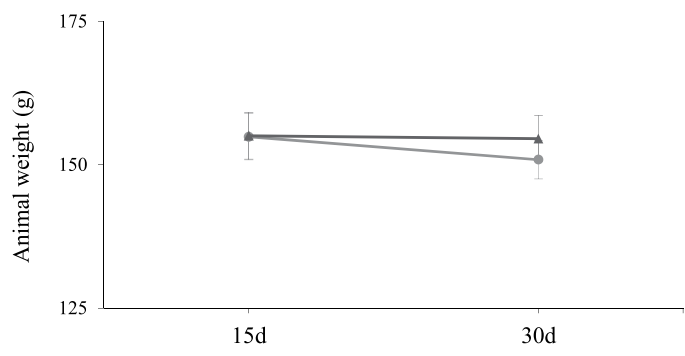

Total cholesterol (TC)

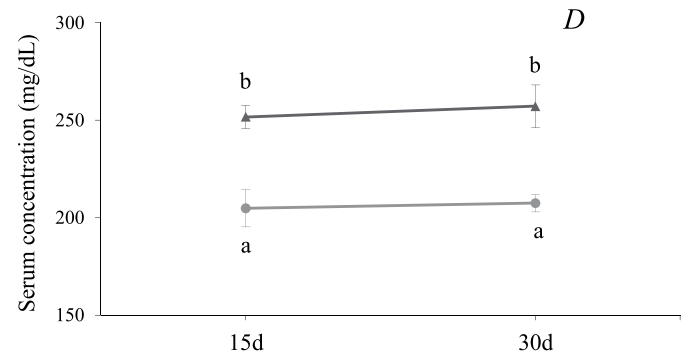

Atherogenic index

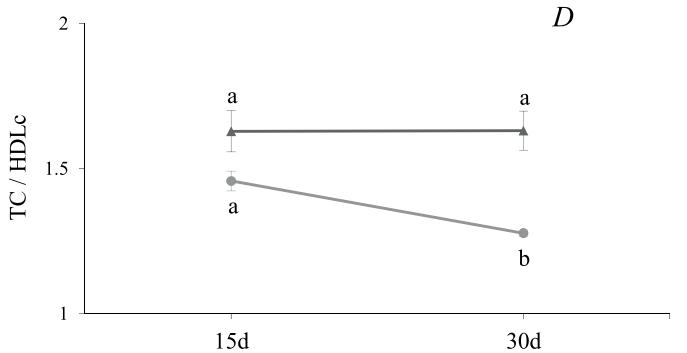

$\rightarrow$ NFD
Liver weight

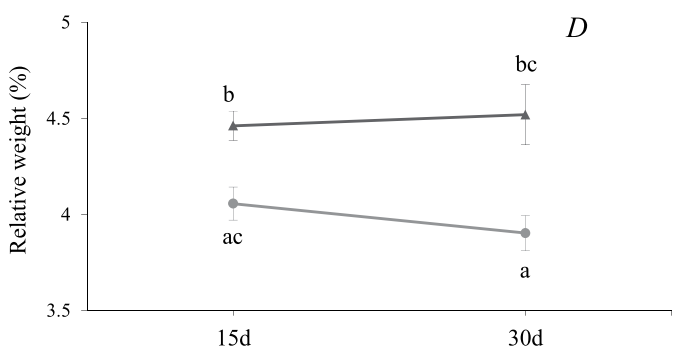

LDLc

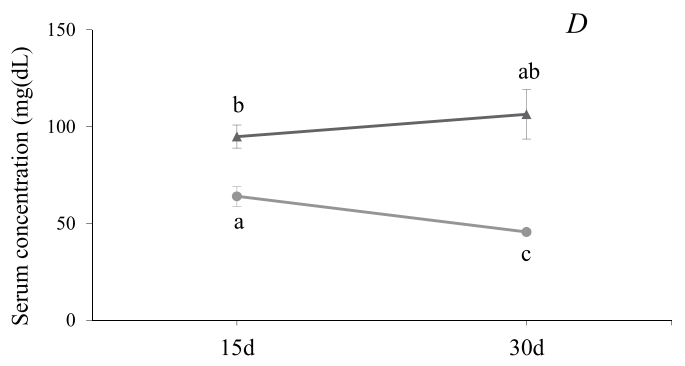

Triglycerides

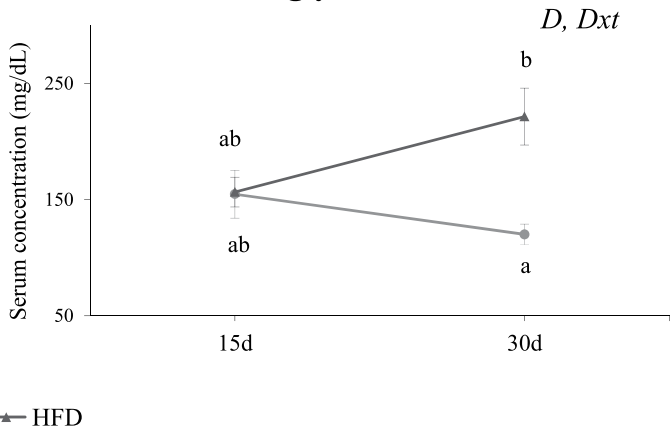

Figure 1. Biological characteristics of the hamsters from the initial study. Adult hamsters were distributed into two groups and fed either a normal-fat diet (NFD) or a high-fat diet (HFD). Half of the animals in each group were sacrificed on day $15(15 \mathrm{~d})$ and the other half were sacrificed on day $30(30 \mathrm{~d})$. Serum parameters were determined after a $6 \mathrm{~h}$ fast. The data are presented as means $\pm \operatorname{SEM}(\mathrm{n}=8$ per group $)$. The statistical comparisons among groups were conducted using two- and one-way ANOVAs. D: the effect of the diet; Dxt: the interaction between diet and time (two-way ANOVA, $\mathrm{p}<0.05$ ). In each figure, different superscript lowercase letters $\left({ }^{a}, b, c\right)$ indicate significant different mean values (one-way ANOVA and Tukey's or Games-Howell post hoc tests, $\mathrm{p}<0.05)$. TC, total cholesterol; LDLc, low-density lipoprotein cholesterol; HDLc, high-density lipoprotein cholesterol.

HFD to the animals for 15d and 30d (Fig. 1). Hamsters fed the HFD showed higher amounts of TC, LDLc and triglycerides in serum, higher values for the atherogenic index (TC/HDLc) and greater relative liver weights than animals fed the NFD ( $\mathrm{p}<0.005$, two-way ANOVA), without alterations in the body weight. However, time did not significantly affect any of these parameters, and only the circulating triglyceride levels were influenced by the interaction between diet and time $(\mathrm{p}=0.008$, two-way ANOVA).

Based on these findings, the state of dyslipidemia in hamsters fed the HFD for $15 \mathrm{~d}$ was too similar to the hamsters fed for $30 \mathrm{~d}$. Nevertheless, the untargeted comparative analysis of the animal serum revealed that two lipid subclasses were differentially modified by HFD feeding according to the length of the treatment (Table 1). On the one hand, the circulating levels of five monoacylglycerophosphocholines, which are usually named lysophosphatidylcholines (Lyso-PCs), were increased in response to HFD intake ( $\mathrm{p}<0.050$, two-way ANOVA), and the increase in all of the identified Lyso-PCs was restricted at 15d. On the other hand, although the diet had an overall effect on lysophosphatidylinositol (18:1) (Lyso-PI (18:1)) ( $\mathrm{p}=0.006$, two-way ANOVA), the serum levels of all of the Lyso-PIs were at least residually increased in the HFD-fed animals compared to NFD-fed animals at 30d $(\mathrm{p}<0.050$, Student's t-test), which was a later response than Lyso-PCs to HFD feeding. Other metabolites, including monoacylglycerols, amino acids and fatty acids such as arachidonic acid, were altered in the HFD-fed groups, but the glycerol-based lysophospholipids (Lyso-PLs) were the most representative lipid class. Concretely, this means that Lyso-PL category was the family of metabolites that exhibited the most numerous and deepest changes in the overall metabolome in this study. Furthermore, Lyso-PLs have been previously described as indicators of 


\begin{tabular}{|c|c|c|c|c|c|}
\hline $\begin{array}{l}\text { Ionization } \\
\text { mode }\end{array}$ & Ion $m / z$ (MS) & $\begin{array}{l}\text { Distinctive } \\
\text { fragments (MS/ } \\
\text { MS) }\end{array}$ & Tentative identification ${ }^{T}$ & Lipid subclass & $\begin{array}{l}\text { Increased with } \\
\text { HFD feeding }\end{array}$ \\
\hline \multirow{5}{*}{$+\mathrm{ESI}$} & $\begin{array}{l}{[\mathrm{M}+\mathrm{H}]^{+}:} \\
524.3743\end{array}$ & $184.1,104.1,60.1$ & Lyso-PC (18:0) & \multirow{5}{*}{ Monoacylglycerophospho cholines } & \multirow{5}{*}{$15 \mathrm{~d}$} \\
\hline & $\begin{array}{l}{[\mathrm{M}+\mathrm{H}]^{+}:} \\
520.3440\end{array}$ & $184.1,104.1,60.1$ & Lyso-PC (18:2) & & \\
\hline & $\begin{array}{l}\mathrm{M}+\mathrm{H}]^{+}: \\
518.3251\end{array}$ & $184.1,104.1,60.1$ & Lyso-PC (18:3) & & \\
\hline & $\begin{array}{l}\mathrm{M}+\mathrm{H}]^{+}: \\
550.3871\end{array}$ & $184.1,104.1,60.1$ & Lyso-PC (20:1) & & \\
\hline & $\begin{array}{l}{[\mathrm{M}+\mathrm{H}]^{+}:} \\
548.3719\end{array}$ & $184.1,104.1,60.1$ & Lyso-PC (20:2) & & \\
\hline \multirow{4}{*}{$-\mathrm{ESI}$} & $\begin{array}{l}{[\mathrm{M}-\mathrm{H}]^{-}:} \\
599.3218\end{array}$ & $419.2,283.3,79.0$ & Lyso-PI (18:0) & \multirow{4}{*}{ Monoacylglycerophospho inositols } & \multirow{4}{*}{$30 \mathrm{~d}$} \\
\hline & $\begin{array}{l}{[\mathrm{M}-\mathrm{H}]^{-}:} \\
597.3060\end{array}$ & $417.2,281.8,79.0$ & Lyso-PI (18:1) & & \\
\hline & $\begin{array}{l}\mathrm{M}-\mathrm{H}]^{-}: \\
621.3060\end{array}$ & $441.0,305.1,79.0$ & Lyso-PI (20:3) & & \\
\hline & $\begin{array}{l}\mathrm{M}-\mathrm{H}]^{-}: \\
619.2906\end{array}$ & $439.0,303.2,79.0$ & Lyso-PI (20:4) & & \\
\hline
\end{tabular}

Table 1. Identification of the serum lysoglycerophospholipids (Lyso-PLs) that were significantly altered by the high-fat diet (HFD) compared to the normal-fat diet (NFD) after 15 and 30 days of feeding in the initial study. ${ }^{t}$ According to the LIPID MAPS ${ }^{32}$ glycerophospholipid nomenclature, the structure of the acyl chain is indicated within parentheses in the 'Headgroup ( $s n 1$ or $s n 2)$ ' format. The headgroup is assumed to be attached to the $s n 3$ position of glycerol. Abbreviations: Lyso-PC, lysophosphatidylcholine; Lyso-PI, lysophosphatidylinositol.

the health status in both animal and humans studies ${ }^{34-36}$. Therefore, taking in consideration all this information and evidence, this first study highlighted the importance of this family of compounds and their potential as suitable candidate biomarkers of dyslipidemia progression.

Evaluation of the involvement of Lyso-PLs in the etiology and early progression of dyslipidemias. We performed a second animal study in which we induced an earlier stage of dyslipidemia by only feeding the animals the HFD for $4 \mathrm{~d}$ to analyze the ability of this class of compounds to serve as biomarkers of dyslipidemia-related diseases. Furthermore, an additional strategy was used to induce hyperlipidemia that was not influenced by the diet in this second study. Therefore, two groups of animals were treated with P407 for $4 \mathrm{~d}$ or $30 \mathrm{~d}$.

Health status of animals with two diverse forms of dyslipidemia. The biometric and serum analyses of the six groups of hamsters are reported in Table 2. At the start of the experiment, all the groups exhibited similar body weights and fat percentages. Total body weight was not affected by the treatments throughout the study, whereas the overall and relative liver weights of the HFD- and P407-treated groups increased, mainly in the animals submitted to the $30 \mathrm{~d}$ treatments, in which significant differences were observed compared with the C-30d group. Nonetheless, only significant effects of the HFD on adiposity were observed, but not P407 administration. HFD-fed animals displayed numerically greater adiposity than the $\mathrm{C}$ groups at both times (Table 2). Although the post hoc analysis only revealed a significant difference in response to HFD intake at $4 \mathrm{~d}$, a residual increase was also noted in the HFD-30d animals compared to the corresponding C group ( $\mathrm{p}=0.014$, Student's t-test). Regarding the biochemical parameters, the pro-dyslipidemic intervention had an overall significant effect by increasing the circulating levels of TC and its main fractions. Although both treatments influenced the TC levels, the increase in the levels of the fraction corresponding to the evaluated lipoproteins is mainly due to HFD intake. According to the post hoc analysis, the TC and LDLc levels in the HFD-fed animals were altered throughout the study, but the response of HDLc was the slowest and increased after 30d of feeding. Conversely, the TC levels in the P407-treated animals increased rapidly but then stabilized and approached the levels of the C-30d group. The atherogenic index, which is calculated as the ratio of TC and HDLc, was also modulated by the pro-dyslipidemic intervention. More specifically, P407-treated animals had increased values of the index compared to each C group beginning at $4 \mathrm{~d}$, whereas significant alterations in response to HFD feeding were only observed at $30 \mathrm{~d}$. On the other hand, sharp increases in the serum triglyceride levels of approximately $200 \%$ and $150 \%$ were produced by $\mathrm{P} 407$ after $4 \mathrm{~d}$ and $30 \mathrm{~d}$ of treatment, respectively, whereas no significant changes were detected between the HFD-fed animals and $\mathrm{C}$ groups. The intervention also induced a significant increase in the serum phospholipid and glucose levels that was more pronounced for phospholipids than for glucose. The increase in the phospholipid levels was generally induced by both pro-dyslipidemic treatments, although the post hoc analysis only revealed a significant difference in the P407-treated animals at $4 \mathrm{~d}$. However, the overall effects on glucose were due to the diet, which showed an increasing trend, particularly at $30 \mathrm{~d}(\mathrm{p}=0.016$, Student's t-test), but P407 did not induce alterations in the glucose levels. Finally, the serum NEFA levels were the only component that was not affected by either treatment throughout the study. 


\begin{tabular}{|c|c|c|c|c|c|c|c|}
\hline & C-4d & HFD-4d & P407-4d & C-30d & HFD-30d & P407-30d & $\begin{array}{l}\text { 2-way } \\
\text { ANOVA }\end{array}$ \\
\hline \multicolumn{8}{|l|}{ Biometric parameters } \\
\hline Initial body weight (g) & $113.83 \pm 2.27$ & $111.66 \pm 2.84$ & $112.59 \pm 3.36$ & $113.94 \pm 1.52$ & $115.45 \pm 1.83$ & $112.53 \pm 1.83$ & \\
\hline Initial fat (\%) & $10.34 \pm 0.74$ & $10.39 \pm 0.72$ & $10.48 \pm 1.15$ & $11.70 \pm 0.41$ & $10.47 \pm 0.61$ & $11.02 \pm 0.50$ & \\
\hline Final body weight (g) & $115.56 \pm 1.97^{\mathrm{a}}$ & $114.87 \pm 2.80^{\mathrm{a}}$ & $114.94 \pm 3.48^{\mathrm{a}}$ & $124.20 \pm 0.96^{\mathrm{ab}}$ & $129.60 \pm 2.38^{\mathrm{b}}$ & $125.80 \pm 1.93^{\mathrm{b}}$ & $t$ \\
\hline Fat increase (\%) & $-0.37 \pm 0.17^{\mathrm{a}}$ & $0.70 \pm 0.24^{\mathrm{b}}$ & $-0.33 \pm 0.17^{\mathrm{a}}$ & $1.47 \pm 0.43^{\mathrm{c}}$ & $3.72 \pm 0.67^{\mathrm{c}}$ & $2.13 \pm 0.35^{\mathrm{c}}$ & $I, t$ \\
\hline Liver weight (\%) & $3.47 \pm 0.12^{\mathrm{a}}$ & $3.65 \pm 0.04^{\mathrm{a}}$ & $3.79 \pm 0.11^{\mathrm{ab}}$ & $3.45 \pm 0.10^{\mathrm{a}}$ & $4.07 \pm 0.09^{\mathrm{b}}$ & $4.04 \pm 0.08^{\mathrm{b}}$ & $I, t$ \\
\hline \multicolumn{8}{|l|}{ Biochemical parameters } \\
\hline $\mathrm{TC}(\mathrm{mg} / \mathrm{dL})$ & $162.36 \pm 6.10^{a}$ & $231.31 \pm 12.16^{\mathrm{bc}}$ & $231.41 \pm 14.09^{\mathrm{bc}}$ & $170.16 \pm 8.00^{\mathrm{a}}$ & $251.30 \pm 8.91^{\mathrm{b}}$ & $200.90 \pm 8.28^{\mathrm{ac}}$ & I, Ixt \\
\hline HDLc (mg/dL) & $73.22 \pm 2.64^{\mathrm{a}}$ & $83.42 \pm 3.60^{\mathrm{ab}}$ & $77.08 \pm 3.31^{\mathrm{a}}$ & $73.70 \pm 5.59^{\mathrm{a}}$ & $94.09 \pm 4.39^{\mathrm{b}}$ & $73.80 \pm 3.12^{\mathrm{a}}$ & $I$ \\
\hline $\operatorname{LDLc}(\mathrm{mg} / \mathrm{dL})$ & $53.94 \pm 1.43^{\mathrm{bc}}$ & $69.82 \pm 4.25^{\mathrm{a}}$ & $65.65 \pm 3.84^{\mathrm{ac}}$ & $47.76 \pm 1.85^{\mathrm{b}}$ & $67.92 \pm 3.01^{\mathrm{a}}$ & $46.92 \pm 4.12^{\mathrm{b}}$ & $I, t, I x t$ \\
\hline $\begin{array}{l}\text { Atherogenic index (TC/ } \\
\text { HDLc) }\end{array}$ & $2.34 \pm 0.11^{\mathrm{ac}}$ & $2.77 \pm 0.09^{\mathrm{ab}}$ & $3.02 \pm 0.17^{\mathrm{b}}$ & $2.25 \pm 0.10^{c}$ & $2.69 \pm 0.08^{\mathrm{ab}}$ & $2.73 \pm 0.07^{\mathrm{ab}}$ & $I$ \\
\hline Triglycerides (mg/dL) & $149.60 \pm 15.78^{\mathrm{a}}$ & $187.16 \pm 15.57^{\mathrm{ac}}$ & $457.21 \pm 58.76^{\text {bd }}$ & $219.82 \pm 11.93^{\text {ce }}$ & $269.28 \pm 16.38^{\mathrm{de}}$ & $533.02 \pm 50.43^{\mathrm{b}}$ & $I, t$ \\
\hline Phospholipids (mg/dL) & $277.96 \pm 11.82^{\mathrm{a}}$ & $319.03 \pm 11.53^{\mathrm{ab}}$ & $356.52 \pm 15.70^{\mathrm{b}}$ & $288.21 \pm 12.88^{\mathrm{ac}}$ & $338.91 \pm 12.50^{\mathrm{ab}}$ & $347.34 \pm 20.14^{\mathrm{bc}}$ & $I$ \\
\hline Glucose (mg/dL) & $231.88 \pm 14.99$ & $253.67 \pm 9.90$ & $209.26 \pm 12.59$ & $200.97 \pm 16.97$ & $254.67 \pm 10.88$ & $220.71 \pm 16.20$ & $I$ \\
\hline NEFAs $(\mathrm{mM})$ & $0.46 \pm 0.09$ & $0.36 \pm 0.05$ & $0.50 \pm 0.06$ & $0.55 \pm 0.10$ & $0.79 \pm 0.16$ & $0.77 \pm 0.14$ & $t$ \\
\hline
\end{tabular}

Table 2. Biometric and serum biochemical parameters measured in each group of hamsters from the second experiment. The hamsters were distributed into six groups, depending on the treatment and the duration of the experiment: control group-4 days (C-4d), diet-induced dyslipidemia-4 days (HFD-4d), pro-dyslipidemic agent-4 days (P407-4d), control group-30 days (C-30d), diet-induced dyslipidemia-30 days (HFD-30d), and pro-dyslipidemic agent-30 days (P407-30d). The diet-induced dyslipidemia was generated by a high-fat diet (HFD) providing $21 \%$ energy as fat and $0.92 \%$ as cholesterol. The pro-dyslipidemic agent was Poloxamer 407 (P407) and the dose was $50 \mathrm{mg} / \mathrm{kg}$ of body weight. P407 animals were fed the control diet and received intraperitoneal injections of the drug every 3 days. Non-P407 hamsters received injections of vehicle $(0.9 \%$ $\mathrm{NaCl}$ ) with equal periodicity. The animals were sacrificed $24 \mathrm{~h}$ after the last administration of $\mathrm{P} 407$ or vehicle and after a $6 \mathrm{~h}$ fast. The concentrations of serum parameters were determined at the end of the experiment. Relative fat and liver weights were determined using the formula $100 *$ (tissue weight/body weight) and expressed as a percentage of the total body weight. Fat increase was calculated as the difference in the relative fat mass at each time point from the initial value for each animal. The data are presented as means \pm SEM $(\mathrm{n}=9-10)$. The statistical comparisons among groups were conducted using two- and one-way ANOVAs. A two-tailed p-value $<0.05$ was considered statistically significant. For the two-way ANOVA: $I$ : the effect of the pro-dyslipidemic intervention (HFD and P407); $t$ : the effect of time; Ixt: the interaction between the two factors. When one-way ANOVA was also significant, the post hoc analysis was used. In each row of the table, different superscript lowercase letters $\left({ }^{\mathrm{a}, \mathrm{b}, \mathrm{c}}\right)$ indicate significant different mean values (Tukey's or Games-Howell tests). TC, total cholesterol; HDLc, high-density lipoprotein cholesterol; LDLc, low-density lipoprotein cholesterol; NEFAs, non-esterified free fatty acids.

In addition, some of the enzymes involved in the extracellular release of Lyso-PLs were evaluated in these hamsters. As shown in Fig. 2, we investigated the serum levels of the enzymes LCAT, sPLA 2 -IIA and PON1. Overall, both treatments induced a decrease in the serum LCAT levels $(\mathrm{p}<0.001$, two-way ANOVA), which was much more evident in the groups treated with $\mathrm{P} 407$ for $4 \mathrm{~d}$ and $30 \mathrm{~d}$ compared with their respective control counterparts (32\% and $14 \%$ decreases at $4 \mathrm{~d}$ and 30d, respectively) (Fig. 2a). As shown in Fig. 2b, the serum sPLA 2 -IIA concentrations exhibited similar responses to both HFD intake and the P407 treatment $(p=0.003$, two-way ANOVA) as the LCAT levels, with a more marked decline in the P407-treated animals than in HFD-fed groups compared with the controls $(24 \%$ and $27 \%$ decreases at $4 \mathrm{~d}$ and $30 \mathrm{~d}$, respectively; $\mathrm{p}=0.032$ and $\mathrm{p}=0.049$, Student's t-test). Although the two-way ANOVA revealed overall effects of the pro-dyslipidemic intervention $(\mathrm{p}=0.005)$ and time $(\mathrm{p}=0.036)$ on the circulating levels of PON1, the pair-wise comparisons did not reveal significant differences at any time when the HFD-fed and the P407-treated animals were compared with the C groups (Fig. 2c). However, the P407-treated animals displayed numerically greater serum PON1 levels than C groups, mainly at $4 \mathrm{~d}(\mathrm{p}=0.031$; Student's t-test).

Based on these results, the P407 treatment induced greater changes in the levels of enzymes involved in the release of Lyso-PLs into the bloodstream than HFD intake.

Relative expression of hepatic genes implicated in the extracellular release of Lyso-PLs. Impaired hepatocyte functions are responsible for alterations in endogenous plasma lipid and lipoprotein levels and the subsequent development of dyslipidemias ${ }^{37}$. For this reason, we evaluated the relative expression levels of some hepatic genes related to the regulation of extracellular Lyso-PL levels (Fig. 3). The pro-dyslipidemic intervention had significant effects on the relative expression levels of the genes encoding the lipase Lipg, the phospholipase Pla2g2a $(\mathrm{p}<0.001)$ and the acyltransferases Lcat $(\mathrm{p}=0.010)$ and Lpcat3 $(\mathrm{p}=0.033)$ (two-way ANOVA). Specifically, the pair-wise comparisons revealed that the mRNA levels of Pla2g2a were significantly higher in P407-4d animals 
$\mathbf{a}$

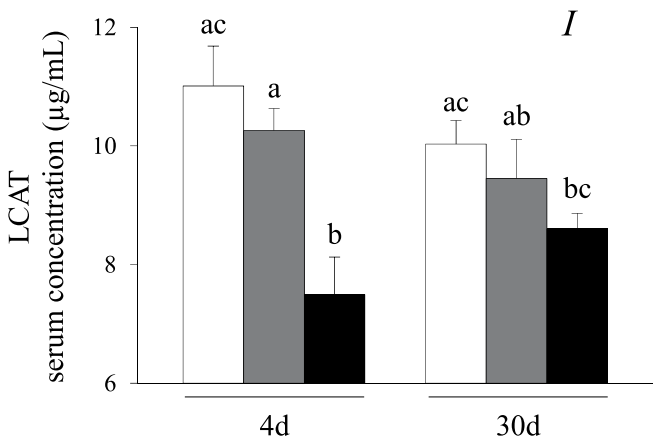

b

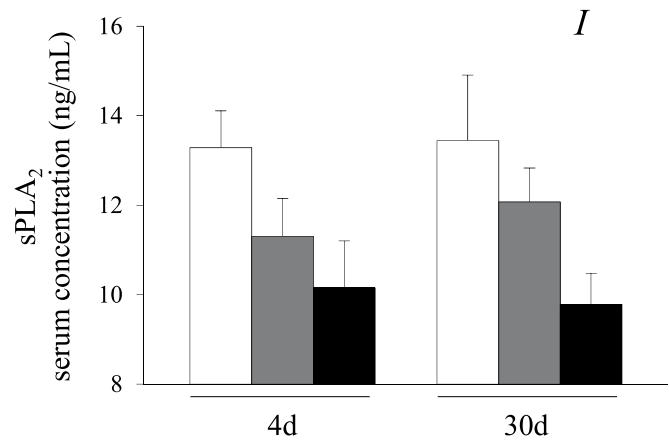

$I, t$ c

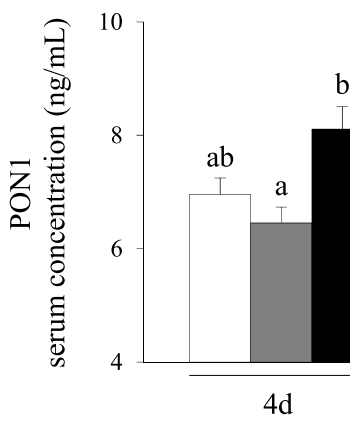

b

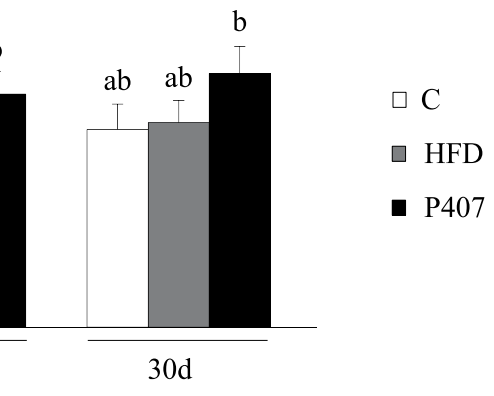

Figure 2. Circulating levels of different enzymes involved in Lyso-PL synthesis in hamsters with different degrees of dyslipidemia. The animals were distributed into three groups: C, group fed a normal-fat diet (NFD); HFD, group fed a high-fat diet; and P407, group fed an NFD and periodically injected with the pro-dyslipidemic agent P407. Half of the animals in each group were sacrificed on day $4(4 \mathrm{~d})$ and the other half were sacrificed on day 30 (30d). The dose of P 407 was $50 \mathrm{mg} / \mathrm{kg}$ every 3 days. Non-P407 hamsters received injections of vehicle $(0.9 \% \mathrm{NaCl})$ with equal periodicity. All animals were sacrificed $24 \mathrm{~h}$ after the last administration of $\mathrm{P} 407$ or vehicle and after a $6 \mathrm{~h}$ fast. The concentrations of serum parameters were determined at the end of the experiment. The data are presented as means $\pm \operatorname{SEM}(n=9-10)$. The statistical comparisons among groups were conducted using two- and one-way ANOVAs. I: the effect of the pro-dyslipidemic intervention (HFD and P407); $t$ : the effect of time (two-way ANOVA, $p<0.05$ ). In each figure, different superscript lowercase letters $(\mathrm{a}, \mathrm{b}, \mathrm{c})$ indicate significant different mean values (one-way ANOVA and Tukey's or Games-Howell post hoc tests, $\mathrm{p}<0.05)$.

than the levels in the C-4d group, and very similar trends were observed for Lipc, Lipg and Lpcat3, showing that the P407-treated animals expressed higher levels of these genes than the $C$ group at $4 \mathrm{~d}(\mathrm{p}<0.050$, Student's $\mathrm{t}$-test). Furthermore, after $30 \mathrm{~d}$ of pharmacological treatment, the P407-30d hamsters displayed a residual increase in Lcat expression and decrease in Abhd3 mRNA levels compared with the C-30d group ( $\mathrm{p}<0.050$, Student's t-test). Moreover, HFD feeding induced alterations in the expression of genes related to hepatic Lyso-PL metabolism, but to a lesser extent than the P407 treatment and, generally, with opposite trends. Thus, compared to the corresponding C group at each time, the expression of Lcat and Lipc was decreased and increased, respectively, in the HFD-4d group ( $\mathrm{p}=0.005$ and $\mathrm{p}=0.049$, Student's t-test), whereas Pla2g2a expression decreased in the HFD-30d hamsters ( $\mathrm{p}=0.002$, Student's t-test). In addition, the levels of the Lipg mRNA were decreased in the HFD-fed animals compared with the control hamsters at both times ( $p=0.024$ and $p=0.001$, Student's $t$-test). No significant differences in the expression of any evaluated gene were observed between the $\mathrm{C}$ groups.

Non-targeted metabolomics of Lyso-PLs and metabolite identification. After confirming that the two pro-dyslipidemic treatments studied here utilize different mechanisms in the liver, we analyzed the circulating metabolome using a non-targeted approach to determine the whole range of altered Lyso-PLs and confirm their suitability as biomarkers of progression and dyslipidemia typology.

After aligning and filtering the molecular entities identified in the six groups of hamsters, the results of the LC-MS/MS analysis showed two characteristic fragmentation patterns that exhibited changes in the dyslipidemic groups (Table 3). Based on the exhaustive study of the fragmentation data, we concluded that both patterns represented Lyso-PL species. In addition, all these Lyso-PLs identified were molecular species containing the acyl chain in sn1-position, which generally is the most common circulating isomer for these metabolites ${ }^{38}$. When collision energies of $10 \mathrm{~V}$ were applied in the positive ionization mode, several daughter ions were generated: a) the loss of the phosphorylethanolamine head group $\left.\left([\mathrm{M}+\mathrm{H}]^{+}-141\right), b\right)$ the loss of water $\left.\left([\mathrm{M}+\mathrm{H}]^{+}-18\right), c\right)$ 
a

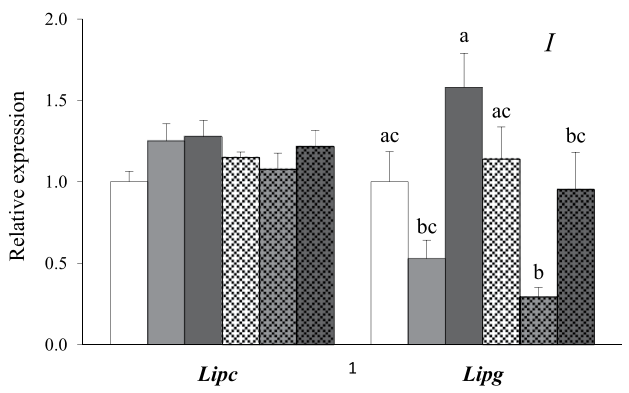

c

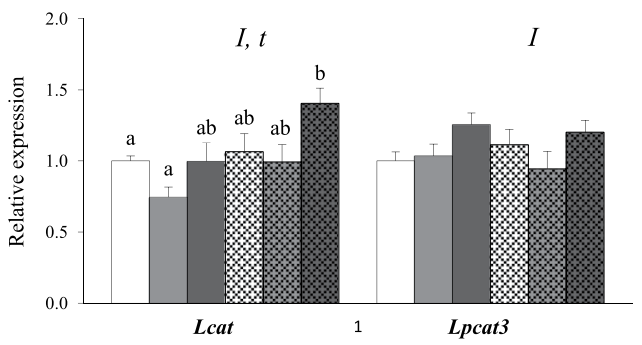

b

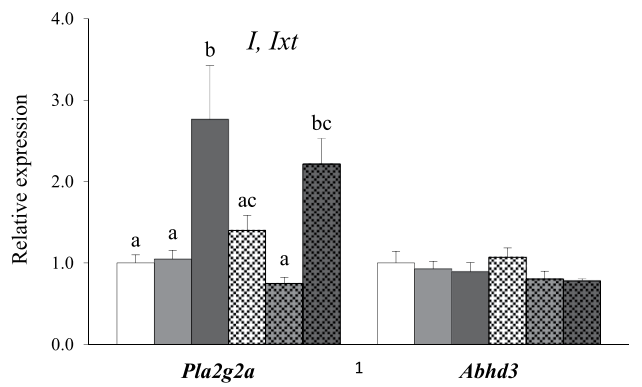

d

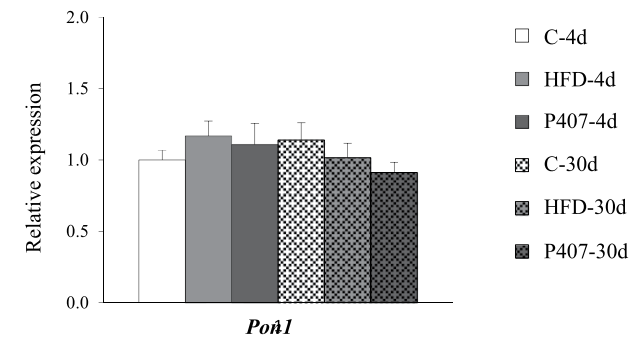

Figure 3. Relative expression levels of hepatic genes implicated in the regulation of the circulating Lyso-PL levels. (a) Lipases: Lipc, hepatic lipase; Lipg, endothelial lipase. (b) Phospholipases: Pla2g2a, phospholipase $\mathrm{A}_{2}$ group IIA; Abhd3, abhydrolase domain-containing 3. (c) Acyltransferases: Lcat, lecithin-cholesterol acyltransferase; Lpcat3, lysophosphatidylcholine acyltransferase 3. (d) Pon1, Paraoxonase 1. The hamsters were assigned to six groups, depending on the pro-dyslipidemic treatment and duration of the experiment: control group-4 days (C-4d), high-fat diet-4 days (HFD-4d), pro-dyslipidemic agent-4 days (P407-4d), control group-30 days (C-30d), high-fat diet-30 days (HFD-30d), and pro-dyslipidemic agent-30 days (P407-30d). Total RNA was isolated from the liver and subjected to qRT-PCR analysis. The relative expression levels were determined using $\beta$-actin as the reference gene and were normalized to the $\mathrm{C}$ - $4 \mathrm{~d}$ group. The data are presented as means $\pm \operatorname{SEM}(\mathrm{n}=9-10)$. The statistical comparisons among groups were conducted using two- and one-way ANOVAs. I: the effect of the intervention (HFD and P407); $t$ : the effect of time; Ixt: the interaction between the two factors (two-way ANOVA, $\mathrm{p}<0.05)$. For each gene, different superscript lowercase letters $(\mathrm{a}, \mathrm{b}, \mathrm{c})$ indicate significant different mean values (one-way ANOVA and Games-Howell post hoc test, $\mathrm{p}<0.05$ ).

\begin{tabular}{|c|c|c|c|c|c|c|}
\hline \multirow{2}{*}{$\begin{array}{l}\text { Neutral } \\
\text { mass }\end{array}$} & \multirow{2}{*}{$\begin{array}{l}\text { RT } \\
(\mathbf{m i n})\end{array}$} & \multicolumn{2}{|c|}{ Collision energy } & \multirow[b]{2}{*}{ Formula (score) ${ }^{Y}$} & \multirow[b]{2}{*}{ Lipid subclass } & \multirow[b]{2}{*}{ Tentative identification } \\
\hline & & $10 \mathrm{~V}$ & $20 \mathrm{~V}$ & & & \\
\hline 475.2661 & 10.85 & $\begin{array}{l}335.3,44.1 \\
458.3\end{array}$ & $44.1,335.3$ & $\mathrm{C}_{23} \mathrm{H}_{42} \mathrm{NO}_{7} \mathrm{P}(86.26)$ & \multirow{6}{*}{$\begin{array}{l}\text { Monoacylglycerophospho } \\
\text { ethanolamines }\end{array}$} & Lyso-PE (18:3) \\
\hline 451.2679 & 10.92 & $\begin{array}{l}311.3,44.1 \\
434.3\end{array}$ & $44.1,311.3$ & $\mathrm{C}_{21} \mathrm{H}_{42} \mathrm{NO}_{7} \mathrm{P}(93.69)$ & & Lyso-PE (16:1) \\
\hline 477.2854 & 11.20 & $\begin{array}{l}337.3,44.1 \\
62.1,460.3 \\
\end{array}$ & $44.1,337.3$ & $\mathrm{C}_{23} \mathrm{H}_{44} \mathrm{NO}_{7} \mathrm{P}(81.06)$ & & Lyso-PE (18:2) \\
\hline 501.2853 & 11.21 & $\begin{array}{l}361.3,44.1 \\
62.1,484.3\end{array}$ & $44.1,361.3$ & $\mathrm{C}_{25} \mathrm{H}_{44} \mathrm{NO}_{7} \mathrm{P}(91.87)$ & & Lyso-PE (20:4) \\
\hline 525.2847 & 11.23 & $\begin{array}{l}385.3,44.1 \\
62.1,508.3 \\
\end{array}$ & $44.1,385.3$ & $\mathrm{C}_{27} \mathrm{H}_{44} \mathrm{NO}_{7} \mathrm{P}(94.58)$ & & Lyso-PE (22:6) \\
\hline 453.2852 & 11.41 & $\begin{array}{l}313.3,44.1 \\
62.1,436.3\end{array}$ & $44.1,313.3$ & $\mathrm{C}_{21} \mathrm{H}_{44} \mathrm{NO}_{7} \mathrm{P}(63.07)$ & & Lyso-PE (16:0) \\
\hline 547.3634 & 11.93 & $184.1,104.1$ & $\begin{array}{l}184.1,104.1 \\
60.1\end{array}$ & $\mathrm{C}_{28} \mathrm{H}_{54} \mathrm{NO}_{7} \mathrm{P}(98.74)$ & \multirow{2}{*}{$\begin{array}{l}\text { Monoacylglycerophospho } \\
\text { cholines }\end{array}$} & Lyso-PC (20:2) \\
\hline 549.3767 & 12.27 & $184.1,104.1$ & $\begin{array}{l}184.1,104.1, \\
60.1\end{array}$ & $\mathrm{C}_{28} \mathrm{H}_{56} \mathrm{NO}_{7} \mathrm{P}(64.24)$ & & Lyso-PC(20:1) \\
\hline
\end{tabular}

Table 3. LC-+ESI-MS/MS identification of the altered Lyso-PLs in the serum of hamsters from the second study. ${ }^{¥}$ The molecular formula score was calculated using the 'Molecular Formula Generator' algorithm included in the MassHunter Qual software. ${ }^{~ A c c o r d i n g ~ t o ~ t h e ~ L I P I D ~ M A P S ~}{ }^{32}$ glycerophospholipid nomenclature, the structure of the acyl chain is indicated within parentheses in the 'Headgroup ( $s n 1$ or $s n 2$ )' format. The headgroup is assumed to be attached at the $s n 3$ position of glycerol. Abbreviations: RT, retention time; Lyso-PE, lysophosphatidylethanolamine; Lyso-PC, lysophosphatidylcholine. 


\begin{tabular}{|c|c|c|c|c|c|c|c|c|c|}
\hline & & \multirow[b]{2}{*}{ C-4d } & \multirow[b]{2}{*}{ HFD-4d } & \multirow[b]{2}{*}{ P407-4d } & \multirow[b]{2}{*}{ C-30d } & \multirow[b]{2}{*}{ HFD-30d } & \multirow[b]{2}{*}{ P407-30d } & \multicolumn{2}{|c|}{ 2-way ANOVA $(I)$} \\
\hline \multicolumn{2}{|c|}{ Compound name } & & & & & & & $p$ & $p$ (Corr) \\
\hline \multirow{6}{*}{ Lyso-PE } & $(20: 4)$ & $-0.33 \pm 0.05^{\mathrm{a}}$ & $0.04 \pm 0.06^{\mathrm{bc}}$ & $0.12 \pm 0.11^{\mathrm{bc}}$ & $-0.18 \pm 0.08^{\mathrm{ac}}$ & $0.18 \pm 0.08^{b}$ & $0.32 \pm 0.10^{\mathrm{b}}$ & $<0.001$ & $<0.001$ \\
\hline & $(18: 2)$ & $-0.17 \pm 0.08^{\mathrm{a}}$ & $-0.06 \pm 0.03^{\mathrm{ac}}$ & $0.28 \pm 0.12^{\mathrm{bc}}$ & $-0.19 \pm 0.10^{\mathrm{a}}$ & $-0.02 \pm 0.09^{\mathrm{ac}}$ & $0.43 \pm 0.14^{\mathrm{b}}$ & $<0.001$ & $<0.001$ \\
\hline & $(18: 3)$ & $-0.10 \pm 0.18^{\mathrm{a}}$ & $-0.22 \pm 0.09^{\mathrm{a}}$ & $0.36 \pm 0.17^{\mathrm{ab}}$ & $-0.23 \pm 0.19^{\mathrm{a}}$ & $-0.30 \pm 0.14^{\mathrm{a}}$ & $0.72 \pm 0.19^{\mathrm{b}}$ & $<0.001$ & $<0.001$ \\
\hline & $(22: 6)$ & $-0.43 \pm 0.10^{\mathrm{a}}$ & $-0.08 \pm 0.10^{\mathrm{ab}}$ & $0.05 \pm 0.17^{\mathrm{ab}}$ & $0.10 \pm 0.11^{\mathrm{b}}$ & $-0.01 \pm 0.08^{\mathrm{ab}}$ & $0.81 \pm 0.11^{\mathrm{c}}$ & $<0.001$ & $<0.001$ \\
\hline & $(16: 1)$ & $-0.26 \pm 0.26^{\mathrm{a}}$ & $-0.25 \pm 0.13^{\mathrm{a}}$ & $0.13 \pm 0.24^{\mathrm{ab}}$ & $0.23 \pm 0.17^{\mathrm{ab}}$ & $-0.20 \pm 0.14^{\mathrm{a}}$ & $0.95 \pm 0.21^{\mathrm{b}}$ & 0.001 & 0.02 \\
\hline & $(16: 0)$ & $-0.14 \pm 0.11^{\mathrm{a}}$ & $-0.13 \pm 0.09^{\mathrm{a}}$ & $0.00 \pm 0.21^{\mathrm{ab}}$ & $0.06 \pm 0.17^{\mathrm{ab}}$ & $-0.34 \pm 0.20^{\mathrm{a}}$ & $0.67 \pm 0.18^{\mathrm{b}}$ & 0.005 & 0.09 \\
\hline \multirow{2}{*}{ Lyso-PC } & $(20: 2)$ & $-0.60 \pm 0.17^{\mathrm{a}}$ & $0.24 \pm 0.10^{\mathrm{bc}}$ & $-0.58 \pm 0.22^{\mathrm{a}}$ & $-0.09 \pm 0.19^{\mathrm{ac}}$ & $0.750 .19^{\mathrm{b}}$ & $-0.16 \pm 0.21^{\text {ac }}$ & $<0.001$ & $<0.001$ \\
\hline & $(20: 1)$ & $-0.08 \pm 0.16$ & $0.35 \pm 0.10$ & $-0.37 \pm 0.22$ & $-0.30 \pm 0.12$ & $0.27 \pm 0.24$ & $-0.29 \pm 0.21$ & 0.003 & 0.08 \\
\hline
\end{tabular}

Table 4. Normalized serum Lyso-PL levels that were significantly altered in the dyslipidemic groups. The levels of each metabolite were transformed to a base-2 logarithm, normalized to the internal standard and to the mean levels of the C-4d samples. The data are presented as the means of the normalized abundances \pm SEM $(n=9-10)$. The two-way ANOVA cut-off point was calculated using the Benjamini-Hochberg correction to avoid false positives. The $\mathrm{p}$-values for the main factor 'intervention' without $(p)$ and with correction $(p(\mathrm{Corr}))$ are shown. I: the effect of the pro-dyslipidemic intervention (HFD and P407 administration). When oneway ANOVA was also significant $(\mathrm{p}<0.05)$, the post hoc analysis was used. In each row of the table, different superscript lowercase letters $\left({ }^{a, b, c}\right)$ indicate significant different mean values (Tukey's test, $\mathrm{p}<0.05$ ).

the ion corresponding to the ethanolamine $\left([\mathrm{M}+\mathrm{H}]^{+}=62\right)$ and its fragment, $\left.\mathrm{d}\right)$ the ethylamine $\left.\left([\mathrm{M}]^{+}=44\right), \mathrm{e}\right)$ the phosphocholine $\left([\mathrm{M}-\mathrm{H}+2 \mathrm{H}]^{+}=184\right)$, and $\left.\mathrm{f}\right)$ choline ions $\left([\mathrm{M}+\mathrm{H}]^{+}=104\right)$. The daughter ions described in $a-d$ are from lysophosphatidylethanolamine (Lyso-PE) species, whereas the daughter ions described in $e-f$ are fragments from the Lyso-PCs identified in the previous study. Published spectra for these lipid subclasses are available in the METLIN database ${ }^{30}$ and Liebisch et al. ${ }^{39}$. The RTs of Lyso-PLs should also be examined. Lyso-PLs with identical acyl chain lengths but different double bond numbers had consistent behaviors. A greater number of double bonds reduced the retention of the particles on the chromatographic column, corresponding to lower RTs.

According to the two-way ANOVA, six Lyso-PEs and two Lyso-PCs exhibited significant changes in the serum of hamsters subjected to HFD feeding and/or P407 treatment for $4 \mathrm{~d}$ or $30 \mathrm{~d}$ (Table 4). However, the post hoc analysis performed on the six groups revealed a different trend that depended on the Lyso-PL class: Lyso-PC levels particularly the Lyso-PC (20:2) levels - were increased in the diet-induced dyslipidemic groups, whereas Lyso-PE abundances mainly increased in the P407-treated groups. Moreover, Lyso-PEs allow us to distinguish the groups with P407-induced dyslipidemia. At 4d, the serum Lyso-PE (18:2) and (20:4) levels were already increased, but the (18:3) and (22:6) levels changed over a longer period. Interestingly, as shown in Table 4 and in Fig. 4, Lyso-PE (20:4) was the unique metabolite that showed a significant increase in response to both the HFD and P407 treatments at $4 \mathrm{~d}$ and at $30 \mathrm{~d}$; in addition, its levels remained constant between both C groups. Therefore, at first glance, Lyso-PE (20:4) may be a putative novel biomarker of dyslipidemia.

\section{Discussion}

Chronic administration of the HFD progressively induced dyslipidemia in adult hamsters after 4, 15 and 30 days of feeding. The biological parameters that first responded to the dietary treatment were the circulating TC and LDLc levels and adiposity index because these parameters increased significantly after $4 \mathrm{~d}$ of the intervention; at $15 \mathrm{~d}$, the consequences of the metabolic disorder started to be observed in the liver weights of the dyslipidemic animals. Finally, the atherogenic index increased after $30 \mathrm{~d}$ of treatment compared to the controls. These findings are compatible with other studies conducted in HFD-fed hamsters ${ }^{20,40-42}$. Consistent with the main objective of the current study, we introduced an additional group of hamsters in which dyslipidemia was induced through periodic injections of P407 instead of by the diet. Despite the low dose used, P407 induced a type of dyslipidemia characterized by a rapid and dramatic increase in the serum triglyceride levels throughout the study, in addition to early hypercholesterolemia (Table 2). The dose-dependent response to P407 was considerably greater for triglycerides than for $\mathrm{TC}^{17}$. Nevertheless, the P407-induced hyperlipidemia addressed in the present study was much milder than the form that has been reported in rodents ${ }^{26,28,29,43}$ and did not exceed the cholesterol levels of the HFD-fed rodents ${ }^{44}$, 5 . Furthermore, the adiposity index and serum levels of HDLc and LDLc were not affected by the chemical treatment, whereas the atherogenic index was altered more quickly than in animals administered the HFD, as an increase was already observed at $4 \mathrm{~d}$. Thus, hamsters that have been periodically treated with P407 are also a good model for studying atherogenic dyslipidemia ${ }^{18,26,29}$. Interestingly, the liver weights of the animals increased after $30 \mathrm{~d}$ in the groups treated with both diet and chemical treatments.

Lyso-PLs were the main metabolites with altered circulating levels that were identified in the dyslipidemia-induced hamsters. These biologically active molecules are involved in a broad range of physiological and pathological processes, such as inflammation, apoptosis, reproduction, immunity, carcinogenesis, angiogenesis and regulation of metabolic diseases ${ }^{46}$. Some of these molecules have been proposed as biomarkers of diabetes ${ }^{47}$, the progression of atherosclerosis ${ }^{48,49}$, obesity $y^{50-52}$ and different types of cancer ${ }^{36,53,54}$ in humans. In addition, in vitro studies using HepG2 cells have shown differences in the patterns of secreted Lyso-PLs under palmitate-induced, noncytotoxic, steatotic conditions ${ }^{51}$. This result is consistent with the serum levels and hepatic 


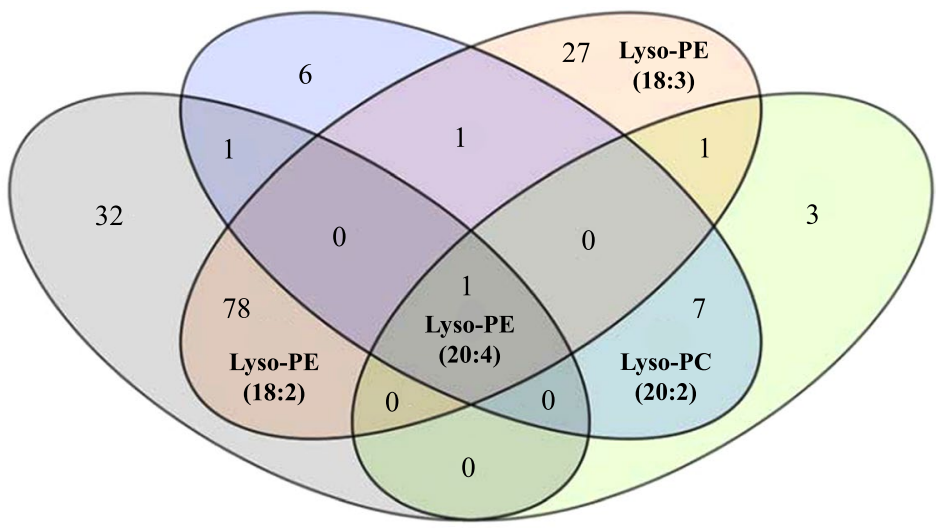

C-4d vs P407-4d $\square$ C-4d vs HFD-4d $\square$ C-30d vs P407-30d $\square$ C-30d vs HFD-30d

Figure 4. Venn diagram displaying the implications of the identified circulating lyso forms in the four comparisons of interest. The statistical comparisons between the six groups $(n=9-10)$ were assessed using twoand one-way ANOVAs. The cut-off point for significance was $\mathrm{p}<0.05$ and for the two-way ANOVA, the p-value of the 'intervention' effect was calculated using the Benjamini-Hochberg correction. The figures indicate the number of significant metabolites for each comparison of interest, which were determined using Tukey's post hoc test. Previously, the metabolites that exhibited significant changes among control groups (C-4d vs C-30d) were discarded. The areas where the ellipses overlap show the significant metabolites, particularly the Lyso-PLs, shared by the specific comparisons. Lyso-PE (20:4) was the only metabolite whose abundance was altered by both diet and the pro-dyslipidemic agent in the short- and long-term assessments.

expressions of the Lyso-PL-related enzymes in our study, many of which were modulated by the pro-dyslipidemic intervention (Figs 2 and 3).

Circulating LCAT is exclusively synthetized by the liver and is an important enzyme involved in the reverse transport of cholesterol and maturation of HDL particles ${ }^{55}$. LCAT fulfils its function by transferring fatty acids from glycerophospholipids to cholesterol, contributing to the removal of cholesterol from the circulation and the release of Lyso-PLs ${ }^{55}$. In this study, P407 administration produced $30 \%$ and $15 \%$ decreases in the circulating LCAT levels after $4 \mathrm{~d}$ and $30 \mathrm{~d}$ of treatment, respectively (Fig. 2a); however, hepatic LCAT expression was only increased at the longer period (Fig. 3c). The increase in the plasma Lyso-PL levels seems to induce the drastic decrease in the LCAT levels after $4 \mathrm{~d}$ of treatment, and the liver attempts to compensate for this shortage by increasing its expression. Moreover, increases in the biological activity of LCAT have been reported in P407-treated rodents ${ }^{17,28}$ as a compensatory mechanism for the high plasma cholesterol loading in these animals. Modifications in exogenous LCAT activity and hepatic mRNA abundance have also been described in hamsters fed different dietary fats ${ }^{42}$, but our results did not show a significant effect on LCAT expression in the diet-induced dyslipidemic animals.

Lipases also contribute to cholesterol homeostasis due to their ability to hydrolyze triglycerides, cholesteryl esters and phospholipids within circulating lipoproteins, mediating important processes involved in the remodeling of these heterogeneous particles ${ }^{55}$. Endothelial lipase (EL) predominantly exerts phospholipase activity and prefers phosphatidylethanolamine as a substrate ${ }^{46,56,57}$. The hydrolysis of this favored substrate leads to the synthesis of Lyso-PEs, which constitute the most representative lipid subclass of our second study. Through its phospholipase activity on HDLc, EL enhances cholesterol removal from peripheral tissues and promotes hepatic accumulation $^{57}$. In this study, both pro-dyslipidemic treatments $(\mathrm{p}<0.001$, two-way ANOVA) modified the hepatic expression of EL (Lipg locus) (Fig. 3a). After 4d, EL expression increased in the P407-treated group and decreased in the HFD-fed hamsters compared to the C-4d group. However, EL expression was also equally influenced by the time and the interaction ( $\mathrm{p}=0.062$, two-way ANOVA), and the levels in both treated groups were decreased by half at day 30 compared to the earlier time points. The inactivation of lipases by P407 has been shown in different rodent models ${ }^{28,58}$ and is related to liver injury ${ }^{18}$. Animal and human studies have revealed that lipases are negative regulators of HDLc levels ${ }^{59,60}$. Moreover, HFD-fed animals showed the lowest EL expression levels in the liver and the highest plasma HDLc levels (Table 2).

sPLA $_{2}$-IIA is a well-known pro-inflammatory enzyme that is involved in arachidonic acid release and eicosanoid production in the secretory compartment ${ }^{61}$. Interestingly, similar to EL, sPLA ${ }_{2}$-IIA has strong activity towards anionic phospholipids, such as phosphatidylethanolamines ${ }^{61}$. Some studies have described a sPLA ${ }_{2}$-mediated progression of liver impairments in rodents when the inducer was a chemical agent ${ }^{62,63}$.

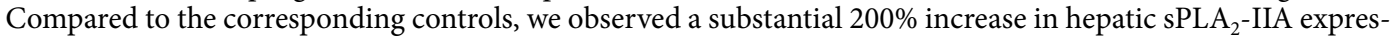
sion (Pla2g2a locus) in the P407-4d animals and 60\% increase ( $\mathrm{p}=0.043$, Student's t-test) in the P407-30d group (Fig. 3b), but not in the animals fed the HFD. This behavior is related to the substantial increase $(\mathrm{p}=0.024$, Student's t-test) in EL expression in the P407-4d hamsters compared to the HFD-fed group (Fig. 3a) because EL has been positively associated with inflammation in rodents and humans ${ }^{57,64}$. The decreased circulating 
sPLA 2 -IIA levels in the P407 groups might be explained by the observation that upon secretion by hepatocytes, the enzyme is rarely into the circulation due to the high rate of synthesis of Lyso-PLs. Based on these findings, animals that have been periodically treated with P407 develop dyslipidemia accompanied by hepatic inflammation, in contrast to the HFD-fed animals.

PON1 is an antioxidant enzyme of primarily hepatic origin that degrades oxidized cholesteryl esters and phospholipids within the lipoprotein $s^{65,66}$ and has been proposed as a suitable non-invasive biomarker for human liver diseases ${ }^{67}$. Liver injury is usually associated with decreased serum activity and hepatic expression but increased circulating PON1 levels ${ }^{68,69}$. In this study, the pair-wise comparisons did not reveal significant differences between the dyslipidemic and control hamsters, but the highest circulating levels were observed in the animals treated with the drug (Fig. 2c) and the largest decrease in hepatic expression of $20 \%$ was observed in the P407-30d group (Fig. 3d). The relation between P407 and PON1 has been described in injected mice, but our results in hamsters were not as clear ${ }^{70}$.

LPCAT3 is responsible for Lyso-PL reacylation and participates in the remodeling of phospholipids and membrane fluidity ${ }^{71}$. The composition of the cell membranes influences the lipid contents in the blood ${ }^{72}$. Mice lacking Lpcat 3 in the liver had lower circulating levels of lipids containing arachidonic acid and inefficiently mobilized triglycerides into very-low density lipoproteins (VLDL), resulting in lipid accumulation in hepatocytes ${ }^{73}$. The P407-treated groups, which were the animals with the highest serum Lyso-PE (20:4) levels, display an early tendency to overexpress Lpcat3 (Fig. 3c), suggesting that hepatic VLDL production may be increased in these animals. In fact, although other authors have reported a shift in the distribution of cholesterol to the VLDL fraction as consequence of P407 administration ${ }^{44}$, we observed an increase in the TC levels in P407-4d hamsters, which did not correspond to either HDLc or LDLc (Table 2).

Thus, we have verified that dyslipidemia was induced by different hepatic mechanisms, depending on the etiology of the disorder $16,27,44,74$. The dietary intervention seems to induce dyslipidemia by inactivating the hepatic genes involved in lipolysis, which induces the liver to sequester lipids ${ }^{27,45,74-77}$; however, oxidative stress and inflammation processes predominate in the animals treated with the chemical agent ${ }^{17,62,64,70}$. Moreover, the development of hepatic steatosis without major inflammation has been described in rodents fed an $\mathrm{HFD}^{74,78}$, whereas a histological examination of livers from P407-treated animals shows defenestration of the sinusoidal endothelium and the accumulation of large foamy Kupffer cells ${ }^{10,43}$. These hepatic alterations are accompanied by the absence of steatosis and are also observed in cirrhosis ${ }^{79}$ and aging ${ }^{80}$. In this context, the Lyso-PLs levels may be used as a biomarker to distinguish typologies of hepatic disorders.

The non-targeted metabolomics evaluation presented in the first study suggested that this class of circulating lipids changes in accordance with the progression of the lipid disorder. The second study seems to confirm their suitability for the evaluation of the dyslipidemia state, showing a joint association of the Lyso-PEs levels with the progression of the disorder induced by the pharmacological treatment; however, the Lyso-PCs appear as specific biomarkers for the dyslipidemia from dietary origin. Among all of the Lyso-PLs, Fig. 4 shows the best putative candidate circulating biomarkers and discards those metabolites that were modified over time in the untreated animals. For their possible application in diagnosis, Lyso-PC (20:2), Lyso-PE (18:2) and Lyso-PE (20:4) were the Lyso-PLs that exhibited the earliest alterations in response to the dyslipidemia. Furthermore, Lyso-PE (20:4) was the unique metabolite that was modulated by both treatments throughout the study.

Due to the key role of lipids in the development and stability of atherosclerotic plaques, dyslipidemia is considerate a primary risk factor for atherosclerosis, which can remain asymptomatic for years. As described above, both types of treatments induced significant increases in the atherogenic index of the hamsters. Interestingly, other authors reported elevated amounts of several Lyso-PCs in atherosclerotic human aorta ${ }^{81}$ and within symptomatic versus asymptomatic carotid plaques ${ }^{48}$. On the other hand, increases in the plasma Lyso-PE (20:4) and (22:6) levels were associated with the occurrence of stable lesions but not unstable plaques ${ }^{49}$.

The elucidation of Lyso-PLs as early biomarkers of pathology is also consistent with recent studies indicating that chronic intake of an HFD dysregulates the plasma and hepatic levels of Lyso-PCs and Lyso-PEs in obese ${ }^{82}$ and non-obese ${ }^{83}$ mice. Consistent with our results, these authors observed altered circulating levels of Lyso-PCs (18:0), (18:2), (18:3) and (20:1) and Lyso-PEs (18:2), (20:4) and (22:6) in HFD-fed rodents. Actually, regarding hepatic disorders, some studies have examined hepatocarcinogenesis progression ${ }^{36}$ and selected certain members of Lyso-PEs as early biomarkers of pathology and altered patterns of Lyso-PCs as plasma indicators to distinguish the most advanced stage, namely the carcinoma phase. Moreover, increases in the serum Lyso-PEs (18:2) and (20:4) levels associated with early liver injury are reversed by fenofibrate therapy, an anti-dyslipidemic agent ${ }^{76,84}$. Therefore, modifications in circulating Lyso-PE and Lyso-PC levels may be related to hepatic disorders and Lyso-PL patterns change with the progression of dyslipidemia-related diseases. Among all the species of Lyso-PLs, Lyso-PE (20:4) seems to possess specific potential as biomarker of the risk of developing lipid pathology.

We concluded that Lyso-PL evaluation could assist with the exhaustive diagnosis and management of lipid disorders since they provide information about the etiology of the dyslipidemia and, furthermore, allow researchers to determine the extent of dyslipidemia. In any case, the omics-based approach used in this study only provided a first approximation of this interesting issue. For instance, it would be necessary to perform a quantitative analysis of the lysophospholipidome by means of the use of an analytical method intended to Lyso-PLs including proper internal standards for these molecules, such as Lyso-PCs (13:0) or (19:0). Despite the abundant evidence in the literature on this topic, we believe that the future studies should be focused on the exhaustive evaluation of the whole family of Lyso-PLs in these animal models. Subsequently, extensive validation in subjects with dyslipidemia-related diseases is required to verify the suitability of the non-invasive biomarkers for use in the population. 


\section{References}

1. Joffres, M., Shields, M., Tremblay, M. S. \& Gorber, S. C. Dyslipidemia prevalence, treatment, control, and awareness in the Canadian Health Measures Survey. Can. J. Public Heal. 104, e252-7 (2013).

2. Steinhagen-Thiessen, E. et al. Dyslipidemia in primary care - prevalence, recognition, treatment and control: data from the German Metabolic and Cardiovascular Risk Project (GEMCAS). Cardiovasc. Diabetol. 7, 31 (2008).

3. Joshi, S. R. et al. Prevalence of dyslipidemia in urban and rural India: The ICMR-INDIAB Study. PLoS One 9, e96808 (2014).

4. Jellinger, P. et al. American Association of Clinical Endocrinologists' Guidelines for management of dyslipidemia and prevention of atherosclerosis. Endocr. Pract. 18, 1-78 (2012).

5. Mahmood, Z., Ahmed, S., Sualeh, M. \& SBZ, M. Hyperlipidemia development and consequences. Med. channel 15, 14-17 (2009).

6. Kuller, L. H. Dietary fat and chronic diseases: Epidemiologic overview. J. Am. Diet. Assoc. 97, S9-S15 (1997).

7. Neuschwander-Tetri, B. A. Hepatic lipotoxicity and the pathogenesis of nonalcoholic steatohepatitis: The central role of nontriglyceride fatty acid metabolites. Hepatology 52, 774-788 (2010).

8. Park, E. J. et al. Dietary and genetic obesity promote liver inflammation and tumorigenesis by enhancing IL-6 and TNF expression. Cell 140, 197-208 (2010).

9. Ahmed, S. M., Clasen, M. E. \& Donnelly, J. E. Management of dyslipidemia in adults. Am. Fam. Physician 57(2192-2204), 2207-8 (1998).

10. Cogger, V. C. et al. Hyperlipidemia and surfactants: the liver sieve is a link. Atherosclerosis 189, 273-81 (2006).

11. Fraser, R., Clark, S. A., Day, W. A. \& Murray, F. E. Nicotine decreases the porosity of the rat liver sieve: a possible mechanism for hypercholesterolaemia. Br. J. Exp. Pathol. 69, 345-50 (1988).

12. Russell, J. C. \& Proctor, S. D. Small animal models of cardiovascular disease: tools for the study of the roles of metabolic syndrome, dyslipidemia, and atherosclerosis. Cardiovasc. Pathol. 15, 318-330 (2006).

13. Dumortier, G., Grossiord, J. L., Agnely, F. \& Chaumeil, J. C. A review of Poloxamer 407 pharmaceutical and pharmacological characteristics. Pharm. Res. 23, 2709-2728 (2006).

14. Wout, Z. et al. Poloxamer 407-mediated changes in plasma cholesterol and triglycerides following intraperitoneal injection to rats. J. Parenter. Sci. Technol. 46, 192-200 (1992).

15. Johnston, T. P. \& Palmer, W. K. Mechanism of poloxamer 407-induced hypertriglyceridemia in the rat. Biochem. Pharmacol. 46, 1037-42 (1993).

16. Johnston, T. P., Nguyen, L. B., Chu, W. A. \& Shefer, S. Potency of select statin drugs in a new mouse model of hyperlipidemia and atherosclerosis. Int. J. Pharm. 229, 75-86 (2001).

17. Johnston, T. P. The P-407-induced murine model of dose-controlled hyperlipidemia and atherosclerosis: a review of findings to date. J. Cardiovasc. Pharmacol. 43, 595-606 (2004).

18. Korolenko, T. A. et al. Early-stage atherosclerosis in poloxamer 407-induced hyperlipidemic mice: pathological features and changes in the lipid composition of serum lipoprotein fractions and subfractions. Lipids Health Dis. 15, 16 (2016).

19. Mawatari, K. et al. Endothelin-1(1-31) levels are increased in atherosclerotic lesions of the thoracic aorta of hypercholesterolemic hamsters. Atherosclerosis 175, 203-212 (2004).

20. Jové, M. et al. Lipidomic and metabolomic analyses reveal potential plasma biomarkers of early atheromatous plaque formation in hamsters. Cardiovasc. Res. 97, 642-652 (2013).

21. Gu, S. et al. Metabonomic profiling of liver metabolites by gas chromatography-mass spectrometry and its application to characterizing hyperlipidemia. Biomed. Chromatogr. 24, 245-252 (2010).

22. Jiang, C. et al. A $1 \mathrm{H}$ NMR-based metabonomic investigation of time-related metabolic trajectories of the plasma, urine and liver extracts of hyperlipidemic hamsters. PLoS One 8, e66786 (2013).

23. Huang, C.-F., Cheng, M.-L., Fan, C.-M., Hong, C.-Y. \& Shiao, M.-S. Nicotinuric acid. Diabetes Care 36, 1729-1731 (2013).

24. Cassol, E. et al. Plasma metabolomics identifies lipid abnormalities linked to markers of inflammation, microbial translocation, and hepatic function in HIV patients receiving protease inhibitors. BMC Infect. Dis. 13, 203 (2013).

25. Jové, M. et al. A plasma metabolomic signature discloses human breast cancer. Oncotarget 8, 19522-19533 (2017).

26. Johnston, T. P. \& Waxman, D. J. The induction of atherogenic dyslipidemia in poloxamer 407-treated mice is not mediated through PPARalpha. J. Pharm. Pharmacol. 60, 753-9 (2008).

27. Palmer, W. K., Emeson, E. E. \& Johnston, T. P. The poloxamer 407-induced hyperlipidemic atherogenic animal model. Atherosclerosis 136, 115-123 (1998).

28. Wasan, K. M., Subramanian, R., Kwong, M., Goldberg, I. J. \& Wright, T. Poloxamer 407 mediated alterations in the activities of enzymes regulating lipid metabolism in rats. J. Pharm. Pharm. Sci. 6, 189-197 (2003).

29. Liu, Q. et al. A dyslipidemia animal model induced by poloxamer 407 in golden hamsters and pilot study on the mechanism. Yao Xue Xue Bao 46, 406-11 (2011).

30. Suizdak, G. \& Abagyan Lab. Scripps Center For Metabolomics and Mass Spectrometry - METLIN. https://metlin.scripps.edu/ (2010).

31. Wishart, D. HMDB: Human Metabolome Database. http://www.hmdb.ca/ (2009).

32. LIPID MAPS. LipidMaps: Nature Lipidomics Gateway. http://www.lipidmaps.org/ (2010).

33. Livak, K. J. \& Schmittgen, T. D. Analysis of relative gene expression data using real- time quantitative PCR and the $2-\Delta \Delta C T$ method. Methods 25, 402-408 (2001).

34. del Bas, J. M. et al. Impairment of lysophospholipid metabolism in obesity: altered plasma profile and desensitization to the modulatory properties of $\mathrm{n}-3$ polyunsaturated fatty acids in a randomized controlled trial. Am. J. Clin. Nutr. 104, 266-279 (2016).

35. Suárez-García, S. et al. Impact of a cafeteria diet and daily physical training on the rat serum metabolome. PLoS One 12, e0171970 (2017).

36. Tan, Y. et al. Metabolomics study of stepwise hepatocarcinogenesis from the model rats to patients: potential biomarkers effective for small hepatocellular carcinoma diagnosis. Mol. Cell. Proteomics 11, M111.010694-M111.010694 (2012).

37. Jiang, J. et al. Influence of liver cancer on lipid and lipoprotein metabolism. Lipids Health Dis. 5, 4 (2006).

38. Suárez-García, S. et al. Development and validation of a UHPLC-ESI-MS/MS method for the simultaneous quantification of mammal lysophosphatidylcholines and lysophosphatidylethanolamines in serum. J. Chromatogr. B 1055, 86-97 (2017).

39. Liebisch, G., Drobnik, W., Lieser, B. \& Schmitz, G. High-throughput quantification of lysophosphatidylcholine by electrospray ionization tandem mass spectrometry. Clin. Chem. 48, 2217-2224 (2002).

40. Caimari, A., Del Bas, J., Crescenti, A. \& Arola, L. Low doses of grape seed procyanidins reduce adiposity and improve the plasma lipid profile in hamsters. Int. J. Obes. 37, 576-583 (2013).

41. Laos, S. et al. Long-term intake of soyabean phytosterols lowers serum TAG and NEFA concentrations, increases bile acid synthesis and protects against fatty liver development in dyslipidaemic hamsters. Br. J. Nutr. 112, 663-673 (2014).

42. Dorfman, S. E., Wang, S., Vega-López, S., Jauhiainen, M. \& Lichtenstein, A. H. Dietary fatty acids and cholesterol differentially modulate HDL cholesterol metabolism in Golden-Syrian hamsters. J. Nutr. 135, 492-8 (2005).

43. Warren, A., Benseler, V., Cogger, V. C., Bertolino, P. \& Le Couteur, D. G. The impact of poloxamer 407 on the ultrastructure of the liver and evidence for clearance by extensive endothelial and kupffer cell endocytosis. Toxicol. Pathol. 39, 390-7 (2011).

44. Johnston, T. P. et al. Potential downregulation of HMG-CoA reductase after prolonged administration of P-407 in C57BL/6 mice. J. Cardiovasc. Pharmacol. 34, 831-842 (1999). 
45. Johnston, T. P. et al. Regression of poloxamer 407-induced atherosclerotic lesions in C57BL/6 mice using atorvastatin. Atherosclerosis 149, 303-313 (2000).

46. Grzelczyk, A. \& Gendaszewska-Darmach, E. Novel bioactive glycerol-based lysophospholipids: New data - New insight into their function. Biochimie 95, 667-679 (2013).

47. Ha, C. Y. et al. The association of specific metabolites of lipid metabolism with markers of oxidative stress, inflammation and arterial stiffness in men with newly diagnosed type 2 diabetes. Clin. Endocrinol. (Oxf). 76, 674-682 (2012).

48. Gonçalves, I. et al. Evidence supporting a key role of Lp-PLA2-generated lysophosphatidylcholine in human atherosclerotic plaque inflammation. Arterioscler. Thromb. Vasc. Biol. 32, 1505-1512 (2012).

49. Meikle, P. J. et al. Plasma lipidomic analysis of stable and unstable coronary artery disease. Arterioscler. Thromb. Vasc. Biol. 31 , 2723-2732 (2011).

50. Moreno-Navarrete, J. M. et al. The L- $\alpha$-Lysophosphatidylinositol/GPR55 system and its potential role in human obesity. Diabetes 61, 281-291 (2012)

51. Del Bas, J. M. et al. Impairment of lysophospholipid metabolism in obesity: altered plasma profile and desensitization to the modulatory properties of n-3 polyunsaturated fatty acids in a randomized controlled trial. Am. J. Clin. Nutr. 104, 266-79 (2016).

52. Kim, J. Y. et al. Metabolic profiling of plasma in overweight/obese and lean men using ultra performance liquid chromatography and Q-TOF Mass spectrometry (UPLC-Q-TOF MS). J. Proteome Res. 9, 4368-4375 (2010).

53. Li, F. et al. Lipid profiling for early diagnosis and progression of colorectal cancer using direct-infusion electrospray ionization Fourier transform ion cyclotron resonance mass spectrometry. Rapid Commun. Mass Spectrom. 27, 24-34 (2013).

54. Sutphen, R. et al. Lysophospholipids are potential biomarkers of ovarian cancer. 13, 1185-1191 (2004).

55. Daniels, T. F. Lipoproteins, cholesterol homeostasis and cardiac health. Int. J. Biol. Sci. 474-488, doi:10.7150/ijbs.5.474 (2009).

56. Chen, S. \& Subbaiah, P. V. Phospholipid and fatty acid specificity of endothelial lipase: potential role of the enzyme in the delivery of docosahexaenoic acid (DHA) to tissues. Biochim. Biophys. Acta 1771, 1319-28 (2007).

57. Annema, W. \& Tietge, U. J. F. Role of hepatic lipase and endothelial lipase in high-density lipoprotein-mediated reverse cholesterol transport. Curr. Atheroscler. Rep. 13, 257-265 (2011).

58. Johnston, T. P. Poloxamer 407 as a general lipase inhibitor: its implications in lipid metabolism and atheroma formation in C57BL/6 mice. J. Pharm. Pharmacol. 62, 1807-12 (2010).

59. Jaye, M. et al. A novel endothelial-derived lipase that modulates HDL metabolism. Nat. Genet. 21, 424-428 (1999).

60. Edmondson, A. C. et al. Loss-of-function variants in endothelial lipase are a cause of elevated HDL cholesterol in humans. J. Clin. Invest. 119, 707-714 (2009).

61. Mallat, Z., Lambeau, G. \& Tedgui, A. Lipoprotein-associated and secreted phospholipases A2 in cardiovascular disease: roles as biological effectors and biomarkers. Circulation 122, 2183-200 (2010).

62. Bhave, V. S., Donthamsetty, S., Latendresse, J. R., Muskhelishvili, L. \& Mehendale, H. M. Secretory phospholipase A2 mediates progression of acute liver injury in the absence of sufficient cyclooxygenase-2. Toxicol. Appl. Pharmacol. 228, 225-238 (2008).

63. Bhave, V. S., Donthamsetty, S., Latendresse, J. R. \& Mehendale, H. M. Inhibition of cyclooxygenase-2 aggravates secretory phospholipase A2-mediated progression of acute liver injury. Toxicol. Appl. Pharmacol. 228, 239-246 (2008).

64. Paradis, M.-E. et al. Endothelial lipase is associated with inflammation in humans. J. Lipid Res. 47, 2808-2813 (2006).

65. Camps, J., Marsillach, J. \& Joven, J. The paraoxonases: role in human diseases and methodological difficulties in measurement. Crit. Rev. Clin. Lab. Sci. 46, 83-106 (2009).

66. Rozenberg, O., Shih, D. M. \& Aviram, M. Human serum paraoxonase 1 decreases macrophage cholesterol biosynthesis: possible role for its phospholipase-A2-like activity and lysophosphatidylcholine formation. Arterioscler. Thromb. Vasc. Biol. 23, 461-467 (2003).

67. Ferré, N. et al. Serum paraoxonase activity: A new additional test for the improved evaluation of chronic liver damage. Clin. Chem. 48, 261-268 (2002).

68. Ferré, N. et al. Paraoxonase-1 is associated with oxidative stress, fibrosis and FAS expression in chronic liver diseases. J. Hepatol. 45, $51-59$ (2006).

69. Marsillach, J. et al. Paraoxonase-1 is related to inflammation, fibrosis and PPAR delta in experimental liver disease. BMC Gastroenterol. 9, 3 (2009).

70. Yasuda, T., Johnston, T. P., Shinohara, M., Inoue, M. \& Ishida, T. The effect of poloxamer 407 on the functional properties of HDL in mice. J. Pharm. Pharmacol. 64, 677-87 (2012).

71. Li, Z. et al. Deficiency in lysophosphatidylcholine acyltransferase 3 reduces plasma levels of lipids by reducing lipid absorption in mice. Gastroenterology 149, 1519-1529 (2015).

72. Rong, X. et al. LXRs regulate ER stress and inflammation through dynamic modulation of membrane phospholipid composition. Cell Metab. 18, 685-697 (2013).

73. Rong, X. et al. Lpcat3-dependent production of arachidonoyl phospholipids is a key determinant of triglyceride secretion. Elife 2015, $1-23$ (2015).

74. Buettner, R. et al. Defining high-fat-diet rat models: metabolic and molecular effects of different fat types. J. Mol. Endocrinol. 36, 485-501 (2006).

75. Rizvi, F. et al. Antidyslipidemic action of fenofibrate in dyslipidemic-diabetic hamster model. Biochem. Biophys. Res. Commun. 305, 215-222 (2003)

76. Srivastava, R. A. K. \& He, S. Anti-hyperlipidemic and insulin sensitizing activities of fenofibrate reduces aortic lipid deposition in hyperlipidemic Golden Syrian hamster. Mol. Cell. Biochem. 345, 197-206 (2010).

77. Cho, A.-S. et al. Chlorogenic acid exhibits anti-obesity property and improves lipid metabolism in high-fat diet-induced-obese mice. Food Chem. Toxicol. 48, 937-943 (2010).

78. Buettner, R., Ottinger, I., Schölmerich, J. \& Bollheimer, L. C. Preserved direct hepatic insulin action in rats with diet-induced hepatic steatosis. Am. J. Physiol. - Endocrinol. Metab. 286, E828-E833 (2004).

79. Mori, T. et al. Defenestration of the sinusoidal endothelial cell in a rat model of cirrhosis. Hepatology 17, 891-897 (1993).

80. Cogger, V. C. et al. Hepatic sinusoidal pseudocapillarization with aging in the non-human primate. Exp. Gerontol. 38, 1101-1107 (2003).

81. Thukkani, A. K. et al. Identification of $\alpha$-chloro fatty aldehydes and unsaturated lysophosphatidylcholine molecular species in human atherosclerotic lesions. Circulation 108, 3128-3133 (2003).

82. Kim, H. J. et al. Metabolomic analysis of livers and serum from high-fat diet induced obese mice. J. Proteome Res. 10, 722-731 (2011).

83. Kim, H. Y. et al. Lysophospholipid profile in serum and liver by high-fat diet and tumor induction in obesity-resistant BALB/c mice. Nutrition 30, 1433-1441 (2014).

84. Shi, X., Yao, D. \& Gosnell, B. a. \& Chen, C. Lipidomic profiling reveals protective function of fatty acid oxidation in cocaine-induced hepatotoxicity. J. Lipid Res. 53, 2318-2330 (2012).

\section{Acknowledgements}

The research leading to these results received funding from the European Union's Seventh Framework Programme "BIOmarkers of Robustness of Metabolic Homeostasis for Nutrigenomics-derived Health CLAIMS Made on Food” Project (BIOCLAIMS) under grant agreement number 244995. SS-G is a recipient of a predoctoral 
fellowship from the Generalitat de Catalunya (2016 FI_B2 00070). The funders had no role in the study design, data collection and analysis, decision to publish, or preparation of the manuscript. We thank Silvia Pijuan, laboratory technician at the Technological Unit of Nutrition and Health (Reus, Spain) for her valuable assistance with the animal studies. We are also grateful to Dr. Maria Rosa Ras and Dr. Pol Herrero, technicians in Centre for Omic Sciences (COS, Reus, Spain), for their support with the LC-MS analyses.

\section{Author Contributions}

L.A. obtained the funding; A.C., J.M.d.B., M.S. and L.A. designed the study; A.C., J.M.d.B. and S.S.-G. conducted the experiments; M.S. and S.S.-G. acquired and analyzed the data; S.S.-G. wrote the main manuscript text and prepared the tables and figures. All authors reviewed the manuscript.

\section{Additional Information}

Supplementary information accompanies this paper at doi:10.1038/s41598-017-10651-0

Competing Interests: The authors declare that they have no competing interests.

Publisher's note: Springer Nature remains neutral with regard to jurisdictional claims in published maps and institutional affiliations.

(c) (i) Open Access This article is licensed under a Creative Commons Attribution 4.0 International License, which permits use, sharing, adaptation, distribution and reproduction in any medium or format, as long as you give appropriate credit to the original author(s) and the source, provide a link to the Creative Commons license, and indicate if changes were made. The images or other third party material in this article are included in the article's Creative Commons license, unless indicated otherwise in a credit line to the material. If material is not included in the article's Creative Commons license and your intended use is not permitted by statutory regulation or exceeds the permitted use, you will need to obtain permission directly from the copyright holder. To view a copy of this license, visit http://creativecommons.org/licenses/by/4.0/.

(C) The Author(s) 2017 